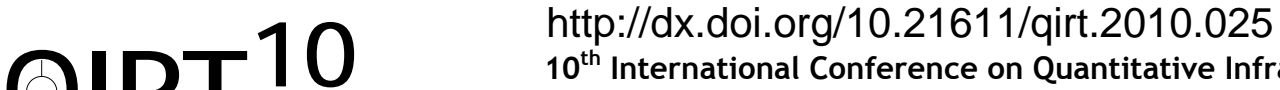 \\ $10^{\text {th }}$ International Conference on Quantitative InfraRed Thermography \\ July 27-30, 2010, Québec (Canada)
}

\section{Quantitative imaging of physical parameters of breakdown sites and avalanche multiplication in solar cells by lock-in thermography}

by O. Breitenstein, J. Bauer, and J.-M. Wagner

\author{
Max Planck Institute of Microstructure Physics, Weinberg 2, 06120 Halle, Germany, breiten@mpi-halle.mpg.de
}

\begin{abstract}
The breakdown behaviour of multicrystalline silicon solar cells under -5 to $-15 \mathrm{~V}$ reverse bias is an important reliability issue for solar modules. Different physical processes may be acting in different breakdown sites. Lock-in thermography (LIT) allows to image local breakdown current densities quantitatively. By performing LIT investigations under different conditions and appropriately combining the results, the temperature coefficient of breakdown sites (in \% current change per $\mathrm{K}$ ) and their slope (in \% current change per $\mathrm{V}$ ) can be imaged quantitatively. By performing special LIT measurements under pulsed illumination, also the local avalanche carrier multiplication factor can be imaged quantitatively.
\end{abstract}

\section{Introduction}

Lock-in thermography (LIT) has been established as a standard method not only for non-destructive testing [1] but also for functional diagnostics of electronic components like solar cells and ICs [2]. For investigating solar cells, several different methods, all based on LIT, have been developed for imaging leakage currents and also other solar cell parameters like, e.g., the series resistance [3]. In recent years the breakdown behaviour of solar cells under reverse bias has gained special interest. The reason is that, under special illumination conditions (local shading), single cells in a typical solar module may be reverse-biased up to $-18 \mathrm{~V}$. If then a strong reverse current is flowing in one position of the cell, this can lead to excessive local heating which may destroy the module. Therefore the breakdown behaviour of solar cells is an important reliability issue for solar modules.

According to established semiconductor theory, silicon solar cells with a base doping concentration of $10^{16} \mathrm{~cm}^{-3}$ should break down at about $-60 \mathrm{~V}$ reverse bias [4]. However, especially for multicrystalline cells, a considerable reverse current is flowing already at $-10 \mathrm{~V}$ and lesser bias. Lock-in thermography is the method of choice for investigating breakdown phenomena in solar cells, since it allows to image both weak and strong local heat sources in these devices with a good spatial resolution [5]. A special advantage of LIT is that its results may easily be interpreted quantitatively, since they depend only on the amount of the local heat dissipation, on the areal heat capacity of the device, and on the lock-in frequency, but not e.g. on the heat conductivity to the surrounding $[2,6]$. For such quantitative investigations the infrared (IR) emissivity needs not be unity. It is sufficient that it is essentially homogeneous across the device area. For the solar cells investigated in this contribution this is the case, since these cells contain a screen-printed Al-paste back contact containing small Al particles and glassy components, which, together with the free carrier absorption in the device, provides a homogeneous IR emissivity of about $\varepsilon=0.66$ [7]. Note that the cells used in [7] were alkaline-etched, leading to relatively large emissivity variations from grain to grain, whereas the cell investigated in this work was acidic-etched, leading to a very homogeneous emissivity. All investigations shown in this contribution were performed on one typical $156 \star 156 \mathrm{~mm}^{2}$ sized solar cell, which was free from ohmic shunts, made industrially from standard multicrystalline solar-grade block-cast silicon material. All LIT investigations were made at a lock-in frequency of $10 \mathrm{~Hz}$ by using a commercial LIT system made by Thermosensorik GmbH (Erlangen).

In this contribution, first the general breakdown behaviour of this cell is demonstrated. Then, after reviewing the basics of quantitative LIT evaluation on thermally thin electronic devices, three LIT methods for quantitative characterization of breakdown sites in solar cells are introduced. Two methods are based on evaluating dark LIT (DLIT) images taken at different temperatures and reverse biases, and the third method is a special LIT method implying pulsed illumination (ILIT) at different reverse biases [8].

\section{The general breakdown behaviour}

Figure 1 (a) shows reverse current-voltage $(I-V)$ characteristics of this cell measured at two temperatures in linear drawing, and (b) shows the characteristic at room temperature in half-logarithmic drawing. We see that a remarkable current flow starts already at $-2 \mathrm{~V}$, and at $-14 \mathrm{~V}$ the reverse current is in the order of $-1 \mathrm{~A}$. The temperature-dependent characteristics in (a) are crossing each other at $-13 \mathrm{~V}$, hence up to $-13 \mathrm{~V}$ the temperature coefficient (TC) of the absolute value of the reverse current is positive (stronger reverse current at higher temperature), and beyond $-13 \mathrm{~V}$ it becomes negative. The semi-logarithmic drawing (b) shows that the current increases exponentially with a certain slope up to $-13 \mathrm{~V}$ reverse bias, but 
beyond this bias the slope (steepness) of the characteristic significantly increases. Already these global $I-V$ results indicate that at different reverse biases there are obviously different breakdown mechanisms active, which differ in the TC and slope of their I-V characteristics. At a reverse bias of $-13 \mathrm{~V}$ there seems to be a transition between different mechanisms. It can be assumed that these different mechanisms are active in different regions of the cell. For confirming this prediction locally imaging investigations are necessary.

a)

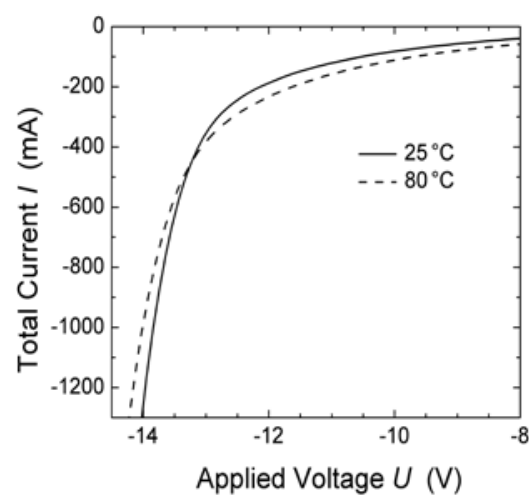

b)

$$
\operatorname{Ir}[\mathrm{mA}]
$$

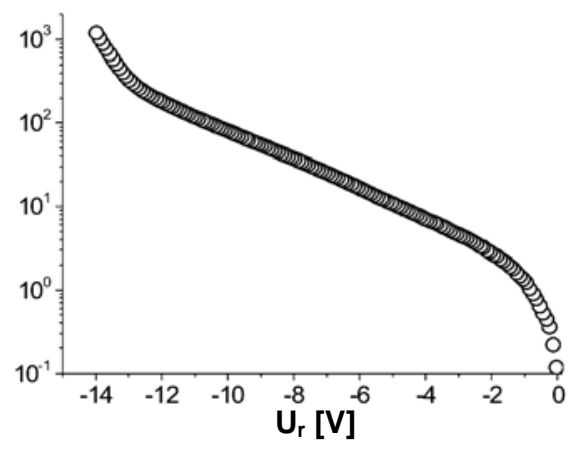

Fig. 1. (a) Reverse current-voltage (I-V) characteristics of the investigated cell in linear drawing at two temperatures, (b) room temperature reverse characteristic in half-logarithmic drawing.

The lock-in thermograms of this cell shown in figure 2 are taken at room temperature under different reverse biases. Note that the scaling ranges of these thermograms are very different. Since the local reverse bias can be assumed to be homogeneous across these solar cells and the emissivity is nearly homogeneous (except of the two horizontally running contact stripes), the brightness in the thermograms in figure 2 can be interpreted to be proportional to the locally flowing reverse current density. Due to the inevitable lateral heat diffusion, point-like heat sources appear spatially extended in the thermograms. Generally, the breakdown current is flowing very inhomogeneously. There is no homogeneous breakdown current. At $-5 \mathrm{~V}$ only a few breakdown sites appear in the area and in the edge region of the cell. At $-12 \mathrm{~V}$ there are many more breakdown sites, which are known to exist at the sites of crystal defects in the silicon material [5]. At - $15 \mathrm{~V}$ these sites are still active, but new breakdown sites appear and become dominant.

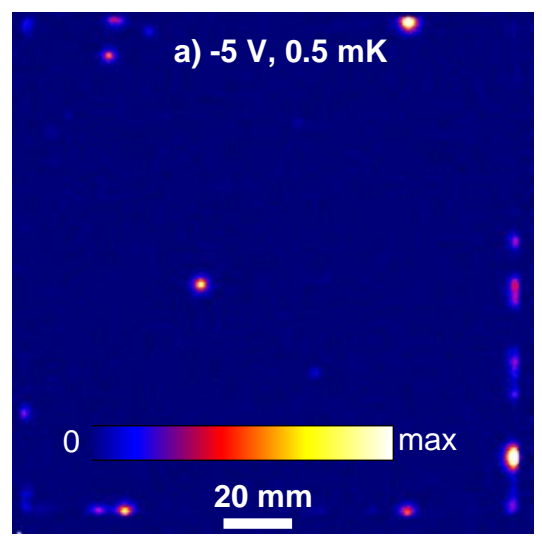

Fig. 2. Lock-in thermograms (amplitude imagr
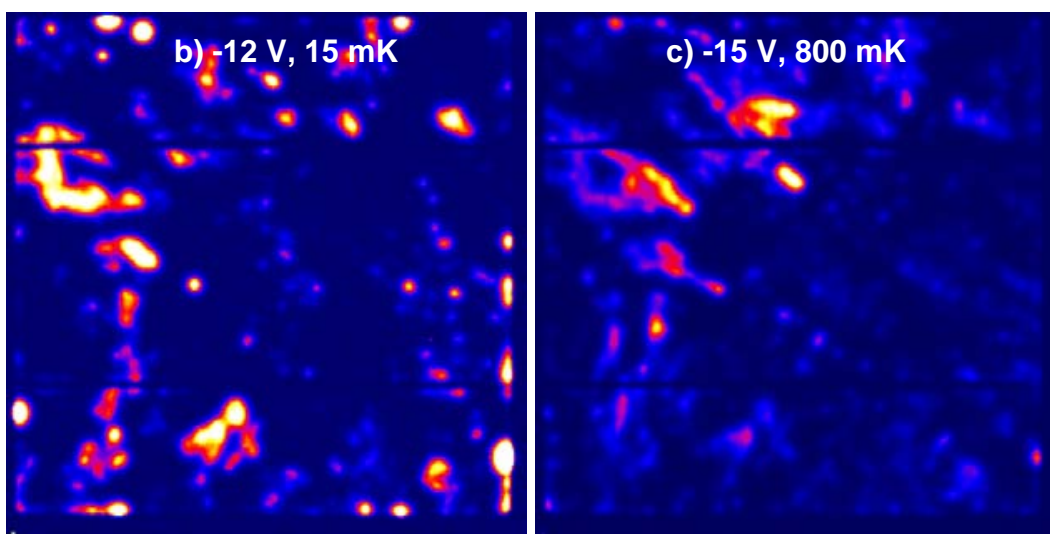
different scaling ranges are indicated.

\section{Basics of quantitative DLIT evaluation}

In contrast to e.g. photothermal LIT investigations, in the investigation of electronic devices the heat is introduced internally by the operation of the device. Therefore DLIT (dark LIT) is used here and the basic task of quantitative evaluation of DLIT results of electronic devices is to convert lock-in thermograms into lateral distributions of dissipated power density $p(x, y)$. In our case the locally flowing current density $J(x, y)$ is the decisive magnitude. For lock-in frequencies in the order of $10 \mathrm{~Hz}$, as they are used here, wafer-based silicon solar cells can be considered as thermally thin, since the thermal diffusion length of about $1.7 \mathrm{~mm}$ clearly exceeds the wafer thickness of about $0.2 \mathrm{~mm}$. It has been shown that under this condition the $-90^{\circ}$ (quadrature) phase component of the DLIT signal is, within the spatial resolution limit of about one diffusion length, 
proportional to the local power dissipation density $[2,6]$. If the emissivity is sufficiently homogeneous, the unknown proportionality factor $C$ between the $-90^{\circ}$ signal $S^{-90}(x, y)$ and the power density $p(x, y)$ (which contains the emissivity as well as several other experimental, apparatus, and device parameters) can be obtained by evaluating the $-90^{\circ}$ signal averaged over the whole device area $A$. If locally

$$
p(x, y)=\operatorname{CS}^{-90^{\circ}}(x, y)
$$

holds, then for the whole cell (where the total dissipated power $P$ is known to be the product of applied bias $V$ and flowing current $I$ )

$$
P=C \int_{\text {area }} S^{-90^{\circ}}(x, y) d x d y
$$

holds. Combining (1) and (2) leads to

$$
p(x, y)=\frac{P}{A<S^{-90^{\circ}}>} S^{-90^{\circ}}(x, y)
$$

with $\left\langle S^{-90^{\circ}}>\right.$ being the $-90^{\circ}$ signal averaged over the device area $A$. If it can be assumed that the voltage $V$ is constant across the device area, the locally flowing current density $J(x, y)$ is $p(x, y)$ divided by the applied voltage $V$, leading to the final result:

$$
J(x, y)=\frac{1}{A<S^{-90^{\circ}}>} S^{-90^{\circ}}(x, y)
$$

In the following, two methods are described which are both based on the application of (4). Note that eq. (4) provides the current density with the same sign as the total current $I$ since $S^{-90^{\circ}}$ is always positive.

\section{TC-DLIT and Slope-DLIT}

It had been shown in Sect. 2 that the different breakdown mechanisms obviously differ not only in their onset voltage but also in the TC and in the slope of their individual $I-V$ characteristics. Therefore methods for imaging the TC and the slope of local breakdown sites are highly desirable. The following two methods are based on the evaluation of a set of $-90^{\circ}$ DLIT images measured at different temperatures $T_{i}$ and biases $V_{i}$. As a first step all these images are converted into current density images $J(x, y, T, V)$ by applying (4). In each position $(x, y)$, the difference between two $J$-values belonging to two different temperatures at fixed bias is a measure of the TC of the current. The difference between two $\mathrm{J}$-values belonging to two different biases at fixed temperature is a measure of the slope of the current. These differences still have to be normalized to the average current density and to the $T$ - and $V$-difference, respectively, for obtaining the relative TC and slope values, independent of the magnitude of the locally flowing current:

$$
\mathrm{TC}(x, y, V)=\frac{2\left[J\left(x, y, T_{2}, V\right)-J\left(x, y, T_{1}, V\right)\right]}{\left(T_{2}-T_{1}\right)\left[J\left(x, y, T_{2}, V\right)+J\left(x, y, T_{1}, V\right)\right]}, \quad \operatorname{Slope}(x, y, T)=\frac{2\left[J\left(x, y, T, V_{2}\right)-J\left(x, y, T, V_{1}\right)\right]}{\left(V_{2}-V_{1}\right)\left|J\left(x, y, T, V_{2}\right)+J\left(x, y, T, V_{1}\right)\right|}
$$

By multiplying (5) by 100 the results are given in units of "\% (current change) / K" and "\% (current change) / V", respectively. In all regions where no breakdown occurs the DLIT signal may be very low. Here, due to the normalization in (5), the TC and the slope signal are dominated by noise. In figure 3 (b) this noise has been cancelled by artificially setting all TC data to zero if the absolute value of $\left[J\left(x, y, T_{2}, V\right)+J\left(x, y, T_{1}, V\right)\right]$ is below a certain threshold value. Since in these methods the slopes are approximated by a difference quotient, care must be taken that the used temperatures and biases are lying sufficiently close together. This is especially important for slope measurements, since the reverse current increases exponentially with bias $V$, as figure 1 (b) shows. Figure 3 shows a typical $-90^{\circ}$ LIT image of a solar cell (a) together with a typical TC image (b) and a slope image (c) derived from two LIT images taken at different temperatures $T$ and reverse biases $V$, respectively. Note that, due to the normalization to the average current densities, the whole region thermally influenced by a local breakdown site shows the corresponding TC and slope signal. The results in figure 3 show that obviously different breakdown mechanisms are acting in different locations of the cell, showing different TCs and slopes. 

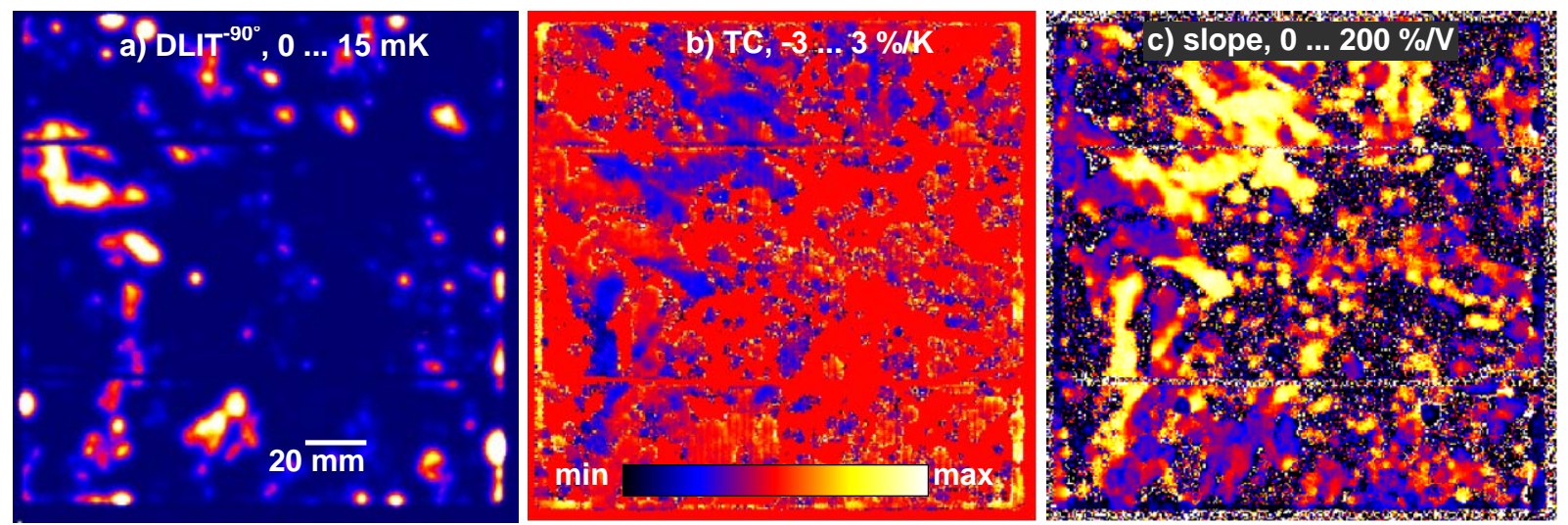

Fig. 3. (a) Lock-in thermogram, (b) temperature coefficient (TC) image, and (c) slope image of a typical solar cell at room temperature and $-13 \mathrm{~V}$ reverse bias. The scaling limits are indicated, the colour scaling bar in (b) holds for all images.

\section{MF-ILIT}

The steep slope and the negative TC of certain regions in figure 3 suggests that avalanche breakdown should be active in these regions. In this case avalanche carrier multiplication should take place. This can be proven by measuring the local avalanche multiplication factor (MF, dimensionless), which is defined as the ratio of the light-induced current density at a certain reverse bias $V$ compared to that measured at a lower bias $V_{\text {low }}$ where no avalanche multiplication takes place. MF = 1 means that there is no avalanche multiplication. The local avalanche multiplication factor can be imaged by applying illuminated lock-in thermography (ILIT). The basic idea is that LIT images are taken at a sufficiently high steady-state voltage, and the solar cell is illuminated homogeneously by pulsed light. Then the local LIT signal should be proportional to the locally flowing photocurrent, which should be increased if avalanche multiplication takes place. Note that for this technique eq. (4) cannot be applied anymore, since under illumination the locally dissipated heat is not simply the product of local bias and current density anymore, see [9]. Moreover, here we have to do with time-independent dark and modulated light-induced current contributions. For the modulated light-induced current density $J_{\text {photo, }}$ which we want to measure, the following relation holds [8]:

$$
J_{\text {photo }}(x, y, V)=\frac{C}{\left(|V|+V_{D}+V_{\text {th }}\right)} S^{-90^{\circ}}(x, y, V)
$$

Here $V_{D}$ is the diffusion voltage of the cell (typically $0.9 \mathrm{~V}$ ) and $V_{\text {th }}$ is the thermalization voltage coming from the fact that the photon energy is above the gap energy of silicon. For illumination with $850 \mathrm{~nm}$ light $V_{\text {th }}=0.35 \mathrm{~V}$ holds. For measuring the voltage-dependent avalanche multiplication factor $\operatorname{MF}(V)$, this ILIT measurement has to be performed twice, once at the voltage $V$ where MF has to be measured, and once at a lower voltage $V_{\text {low }}$ where definitely no avalanche multiplication takes place yet. Finally, MF is the ratio of these two photocurrents according to (6), which cancels the unknown proportionality factor $C$ :

$$
\operatorname{MF}(x, y, V)=\frac{\left(\left|V_{\text {low }}\right|+V_{\mathrm{D}}+V_{\text {th }}\right) S^{-90^{\circ}}(x, y, V)}{\left(|V|+V_{\mathrm{D}}+V_{\text {th }}\right) S^{-90^{\circ}}\left(x, y, V_{\text {low }}\right)}
$$

Figure 4 shows two $-90^{\circ}$ ILIT images measured at room temperature at constant reverse biases of $V_{\text {low }}=-13 \mathrm{~V}$ and $V=-14.5 \mathrm{~V}$, respectively, and pulsed homogeneous illumination, together with the resulting avalanche multiplication factor (MF) image. At -13 V the ILIT image is still looking quite homogeneous, hence at $-13 \vee$ the photocurrent is flowing essentially homogeneously. At this voltage there is no indication of avalanche multiplication yet. At $-14.5 \mathrm{~V}$, however, clear local maxima appear which indicate avalanche multiplication. Indeed, the regions showing negative TC and high slope in figure 1 also show strongest avalanche multiplication as indicated in figure 4 (c). 

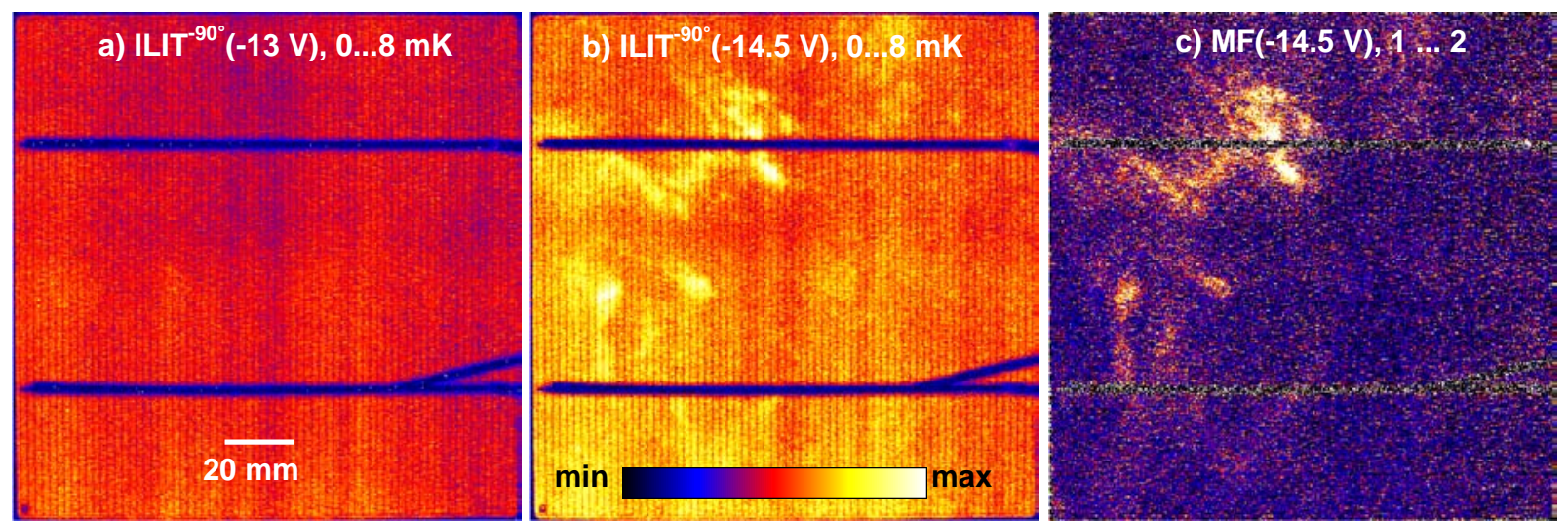

Fig. 4. Illuminated lock-in thermography (ILIT) images at (a) -13 V and (b)-14.5 V, (c) avalanche multiplication factor derived from (a) and (b). The colour scaling bar in (b) holds for all images.

\section{Conclusions}

Already as a simple imaging technique, lock-in thermography allows to perform the bias-dependent imaging of breakdown sites in solar cells, which allows to draw important conclusions as to the physical processes being responsible for the breakdown. It was shown in this contribution that special LIT techniques may be applied to image physical parameters of breakdown sites quantitatively. TC-DLIT allows to image the normalized temperature coefficient and slope-DLIT the normalized slope (steepness) of a breakdown current. The MF-ILIT technique allows to image the bias-dependent local avalanche multiplication factor quantitatively. Local avalanche multiplication is a clear proof that certain breakdown sites are caused by the avalanche effect. Indeed, it has been shown that in these regions etch pits are present who locally increase the electric field so strongly that avalanche multiplication may take place at reverse biases as low as -14 V [10].

\section{REFERENCES}

[1] Maldague X.P.V., "Theory and Practice of Infrared Technology for Nondestructive Testing", Wiley, New York (USA), 684p., 2001.

[2] Breitenstein O. and Langenkamp M., "Lock-in Thermography - Basics and Use for Functional Diagnostics of Electronic Components”, Springer, Berlin, Heidelberg (Germany), 193 p., 2003 (new edition in 2010).

[3] Breitenstein O., Rakotoniaina J.P., van der Heide A.S.H., and Carstensen J., "Series Resistance Imaging in Solar Cells by Lock-in Thermography", Prog. Photovolt: Res. Appl. 13, p. 645-660, 2005.

[4] Sze S.M. and Gibbons G., "Avalanche Breakdown Voltages of Abrupt and Linearly Graded p-n Junctions in Ge, Si, GaAs, and GaP", Appl. Phys. Lett. 8, p. 111-113, 1966.

[5] Wagner J.-M., Bauer J., Lotnyk A., and Breitenstein O., "Pre-breakdown mechanisms in multicrystalline silicon solar cells", Proc. 23rd Eur. Photovoltaic Solar Energy Conference (PVSEC), Valencia, Spain, September 1-5, 2008, pp. 1164-1168

[6] Breitenstein O., Rakotoniaina J.P., and Al Rifai M.H., "Quantitative Evaluation of Shunts in Solar Cells by Lock-in Thermography", Prog. Photovolt: Res. Appl. 11, p. 515-526, 2003.

[7] Kaminski, A., Jouglar J., Volle C., Natalizio S., Vuillermoz P.L., and Laugier A., "Non-destructive Characterisation of Defects in Devices Using Infrared Thermography", Microelectronics Journal 30, p. 1137-1140, 1999.

[8] Breitenstein O., Bauer J., Wagner J.-M., and Lotnyk A., "Imaging Physical Parameters of Pre-Breakdown Sites by Lock-in Thermography Techniques", Prog. Photovolt: Res. Appl. 16, p. 679-685, 2008.

[9] Breitenstein O. and Rakotoniaina J.P., "Electrothermal Simulation of a Defect in a Solar Cell", J. Appl. Phys. 97, 074905, 2005.

[10] Bauer J., Wagner J.-M., Lotnyk A. Blumtritt H., Lim B., Schmidt J., and Breitenstein O., "Hot Spots in Multicrystalline Silicon Solar Cells: Avalanche Breakdown due to Etch Pits", Phys. Status Solidi RRL 3, p. 40-42, 2009. 